\title{
Norois
}

Environnement, aménagement, société

$199 \mid 2006 / 2$

Canada : immigration, espaces urbanisés, parcs nationaux, loisir patrimonial

\section{Espaces urbanisés et parcs nationaux : le défi de la gestion des espaces urbanisés dans les parcs nationaux de l'Ouest canadien}

Urban spaces and national parks: the challenge of urban spaces management in western canadian national parks

\section{Stéphane Héritier}

\section{(2) OpenEdition}

\section{Journals}

\section{Édition électronique}

URL : https://journals.openedition.org/norois/1928

DOI : $10.4000 /$ norois. 1928

ISBN : 978-2-7535-1547-5

ISSN : $1760-8546$

\section{Éditeur}

Presses universitaires de Rennes

Édition imprimée

Date de publication : 1 juin 2006

Pagination : 61-76

ISBN : 978-2-7535-0332-8

ISSN : 0029-182X

\section{Référence électronique}

Stéphane Héritier, «Espaces urbanisés et parcs nationaux : le défı de la gestion des espaces

urbanisés dans les parcs nationaux de l'Ouest canadien », Norois [En ligne], 199 | 2006/2, mis en ligne le 13 décembre 2008, consulté le 14 janvier 2022. URL : http://journals.openedition.org/norois/1928 DOI : https://doi.org/10.4000/norois. 1928 


\title{
ESPACES URBANISÉS ET PARCS NATIONAUX :
}

\section{LE DÉFI DE LA GESTION DES ESPACES URBANISÉS \\ DANS LES PARCS NATIONAUX DE L'OUEST CANADIEN}

\author{
STÉPHANE HÉRITIER \\ Université JEAN-MONNET \\ Centre de Recherches sur L’Environnement et L'Aménagement, UMR 5600 - CNRS \\ stephane.heritier@univ-st-etienne.fr
}

\begin{abstract}
RÉSUMÉ
Depuis leur création, six parcs nationaux canadiens disposent de centres d'accueil destinés à répondre aux besoins élémentaires des visiteurs (hébergement, alimentation, approvisionnement, sécurité). Connaissant un succès croissant pendant le XXe siècle, ces "sites urbains " sont devenus des collectivités parfois très peuplées et couvrant des espaces relativement importants. Certaines d'entre elles ont même acquis un statut municipal tandis que les autres collectivités sont tonjours sous la tutelle de Parcs Canada. La proportion de résidents et le nombre élevé de visiteurs pendant l'été contribuent à faire de ces centres d'accueil de véritables villes temporaires, rencontrant comme celles-ci tous les problèmes urbains: planification urbaine, gestion des eaux usées, réseaux, voirie, etc. Cet article cherche à saisir la complexité de la situation et de montrer les mécanismes de gestions originaux qui doivent être appliqués à des organismes de type urbain au cour d'espaces préservés.
\end{abstract}

Mots CLÉS : Aménagement - Canada - Collectivité - Environnement - Parcs nationaux - Politiques publiques - Tourisme - Ville.

\section{ABSTRACT}

\section{Urban spaces and national parks: the challenge of urban spaces management in western canadian national parks}

Since the beginning of the national park movement in Canada, some visitor centres were established within six national parks for visitors immediate such as food, supply, lodging and health care. As National Parks became more and more popular those townsites have quickly grown, even including permanent residents. In recent years, some of those communities became Municipalities, creating a unique situation within the Canadian Park System. Considering the number of permanent residents and the number of tourists during the summer season, these communities are facing urban development issues: urban planning, water, sewer and garbage concerns, land use zoning, etc. This paper intends to present the uniqueness of the Canadian Parks situation and to show the way the communities' stakeholders deal with urban issues within protected spaces.

KEY WORDS : Canada - Community - Environment - National Parks - Public Policy - Tourism - Townsite - Urban Development. 
D’une manière générale, les aires protégées comptent peu d'espaces très développés. On s'attend ainsi à ce qu'elles soient exemptes de développement urbain et généralement « le monde rural est aux portes du parc» (Richez, 1992). Au Canada, l'histoire et la mise en place du réseau de parcs nationaux ${ }^{1}$ constituent une originalité stimulante dans la mesure où, au cœur même des espaces protégés, il existe aujourd'hui des secteurs présentant des caractéristiques urbaines - en terme de fonctions, d'organisation du bâti - et dotés des statuts administratifs distincts. Le nombre de collectivités ${ }^{2}$ (communities) - urbaines par les services qu'elles procurent - est limité dans les parcs canadiens, puisque sept villes et collectivités sont recensées dans six parcs, tous situés dans les provinces des Prairies et des montagnes de l'Ouest (fig. 1).

Dans la plupart des cas, ces 'sites urbains' préexistaient aux parcs. Lorsque le gouvernement fédéral récupéra les terres, les rares résidents ne furent pas expulsés, mais ils bénéficièrent de baux peu coûteux et de longue durée. Par la suite, le développement commercial et touristique favorisa l'installation d'un peuplement permanent, en partie encouragé jusque dans les années 1960 (Lothian, 1977, 1979; McKim, 1970 ; Lowry, 1994).

Aujourd'hui, Parcs Canada est directement responsable de la gestion de cinq collectivités ou «visitor centre - centres de visiteurs » de taille inégale (tableau 1): Field, dans le PN Yoho (ColombieBritannique), Lake Louise dans le PN Banff (Alberta), Waterton dans le PN des Lacs Waterton (Alberta), Wasagaming dans le PN Riding Mountain (Manitoba), Waskesiu dans le PN Prince Albert (Saskatchewan) et l'Agence fédérale est associée à la gestion de deux municipalités : Banff et Jasper (Alberta). Parmi ces collectivités et centres d'accueils gérés par l'administration fédérale, les villes de Banff et de Jasper occupent une situation différente. Banff dispose d'un gouvernement municipal autonome depuis 1990, dans le cadre d'un accord entre la province et l'État fédéral; Jasper est dans une situation voisine depuis avril 2002.

Cet article s'intéressera au défi que représente la gestion des espaces urbanisés (villes et collectivités) dans les parcs nationaux de l'Ouest canadien. Intégrés au cœur d'espaces protégés, de la grande nature sauvage (wilderness) nord-américaine, les « sites urbains » constituent ce qu'un géographe canadien qualifiait de « hiatus » (Scace, 1969) et ce que les environnementalistes canadiens les moins virulents définissent comme une incongruité. Quelle que soit la variété des points de vue concernant le développement de ces sites urbains dans les parcs, force est de constater que leur ancienneté, leur taille, leurs fonctions et les revenus qu'ils contribuent à produire en font des objets géographiques particulièrement intéressants. Très fréquentés depuis plusieurs décennies, ils sont devenus les lieux d'enjeux à différents niveaux et à différentes échelles.

Dans cette perspective, il importe de comprendre la manière dont ces communautés s'intègrent dans l'espace parc. Par ailleurs, comment se mettent en place et comment fonctionnent les actions de gestion? Quels sont les principaux problèmes issus de la forte fréquentation estivale et de la présence d'une vie permanente dans ces espaces protégés? À quelles formes de concurrences d'usage, spatiales et temporelles, assiste-t-on et comment les pouvoirs publics y répondent-ils ? Cette réflexion sera guidée en abordant les dynamiques propres à ces centres d'accueil et leurs modalités de gestion, la recherche d'un équilibre s'avérant à la fois complexe, délicate, et à l'origine de tensions et de controverses.

1. Il compte 41 parcs nationaux en 2005.

2. Dans le vocabulaire employé dans les documents officiels des parcs, les termes anglais de visitor centre, townsite et community sont utilisés de manière interchangeable (Environment Canada, 1988). Ces termes désignent les regroupements concentrés de bâtiments, inégalement peuplés et dotés de fonctions urbaines et touristiques. Ces termes excluent, par exemple, les stations de ski ou les secteurs d'activité économique privés désignés sous le terme de logements commerciaux périphériques (sur ce point, se reporter à S. Héritier, 2002). 


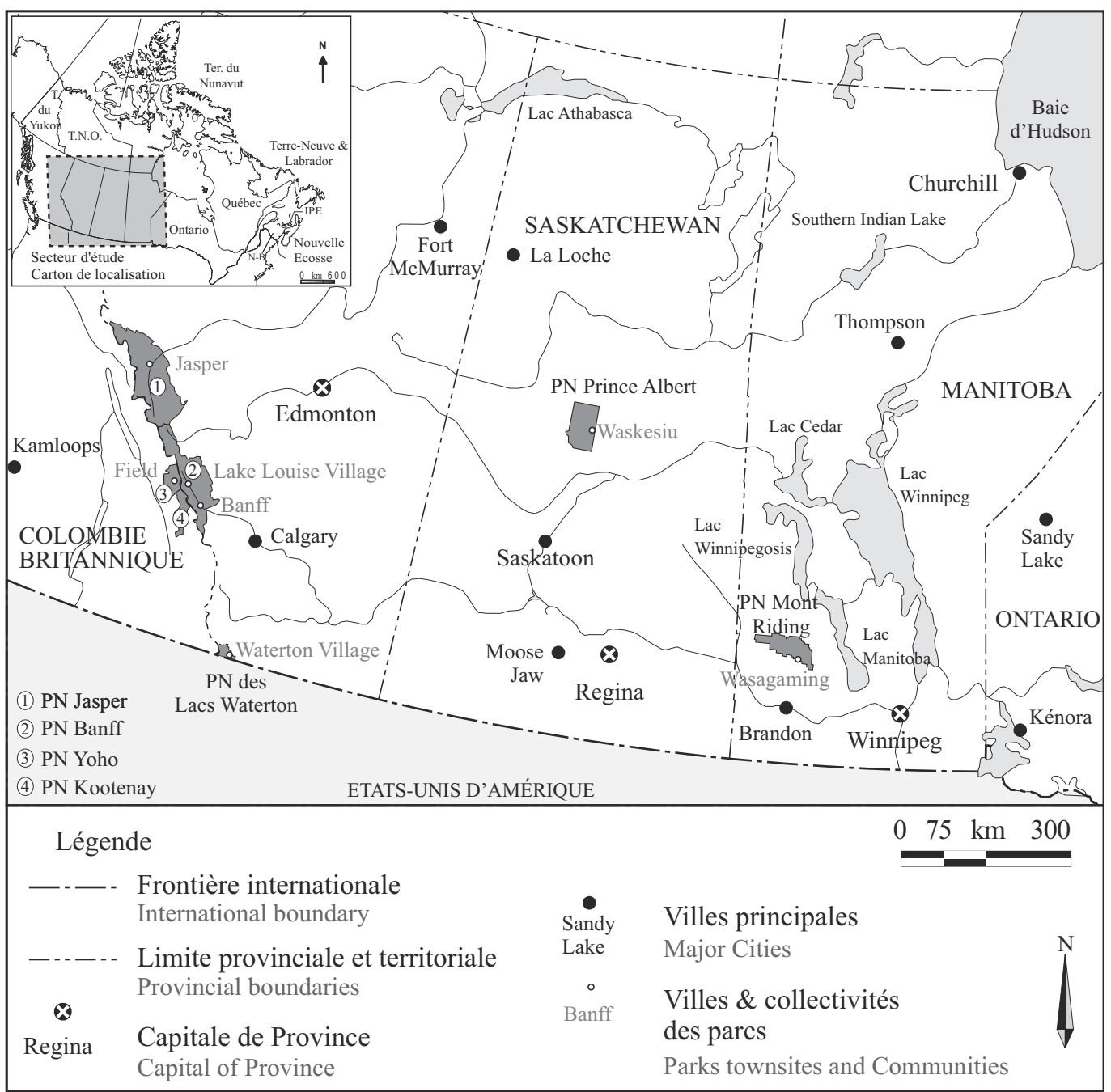

Figure 1 : Villes et collectivités de parcs dans l'Ouest canadien Townsites and Communities in Canadian Western National Parks

\section{Des collectivités entre dynamiques urbaines et touristiques et la protection de la nature}

Les parcs nationaux du Canada sont généralement constitués d'espaces de nature sauvage (wilderness) dont la fonction est double : protéger des écosystèmes spécifiques et accueillir des visiteurs tout à la fois. Ces missions doivent être réalisées dans le cadre de la réglementation propre aux parcs nationaux. En effet, la Loi sur les parcs nationaux dédie les parcs nationaux au « peuple canadien afin que celui-ci puisse les utiliser pour son plaisir et l'enrichissement de ses connaissances $[\ldots]$; ils doivent être entretenus et utilisés de façon à rester intacts pour les générations futures » (Parcs Canada, 2000-J). 
STÉPHANE HÉRITIER

Ainsi, dans certains parcs, les centres d'accueils furent progressivement dotés de fonctions urbaines. Le succès rencontré par ces espaces récréatifs particulièrement recherchés a eu pour effet de concentrer les enjeux dans ces espaces aux dimensions limitées.

\section{CréATION ET DÉVELOPPEMENT DES CENTRES D’ACCUEIL Dans les Parcs Nationaux Canadiens}

Depuis l'origine des parcs nationaux, le gouvernement fédéral a misé sur la polyvalence des fonctions, le rôle des centres d'accueil et, progressivement, sur une mission pédagogique : ils ont servi successivement de support au développement des compagnies ferroviaires nationales puis des secteurs miniers et forestiers pour se concentrer, depuis les années 1970, sur une conception mixte - considérée comme paradoxale - combinant la protection de l'environnement et le développement touristique. Deux décennies plus tard, pendant les années du mandat ministériel de Sheila Copps (ministre du Patrimoine, en charge des Parcs Nationaux de 1997 à 2003), ces espaces furent gérés dans la perspective de la protection des écosystèmes : la notion d'intégrité écologique 3 (Richez, 2000b) étant étroitement associée à celle de développement durable.

D’une manière générale, il est utile de rappeler que la présence humaine est temporaire, à quelques exceptions près. Si des installations pérennes existent, leur usage est souvent saisonnier. Par ailleurs, les implantations permanentes ont tendance, depuis plusieurs années, à être limitées. Toutefois, six parcs nationaux sont dotés de centres d'accueil, de dimension et d'importance variables, gérées par Parcs Canada jusqu'en 1990. Aujourd'hui, il convient de distinguer les villes des centres d'accueil, qui diffèrent par leur taille et les fonctions qui y sont associées.

Dès la création des parcs nationaux Banff (1885) et Jasper (1907), une population pionnière s'est installée à proximité des bâtiments ferroviaires des compagnies Canadian Pacific (Banff) et Canadian National (à Jasper). Ces établissements ont créé des villages qui ont rapidement diversifié leurs fonctions pour devenir des centres d'accueil pour les voyageurs des compagnies ferroviaires, avant d'accueillir un nombre grandissant de visiteurs motorisés. Devenus stations touristiques et centres administratifs, ces petits noyaux de peuplement gagnèrent des effectifs et furent dotés de fonctions urbaines. Assez rapidement, Parcs Canada se trouva dans la situation curieuse de gérer des « centres d'accueil » qui présentaient des caractéristiques urbaines très marquées. Dans cette perspective, et pour répondre aux demandes des résidents et des acteurs de la collectivité, Banff fut dotée d'un gouvernement municipal autonome, régie par le droit provincial albertain. Douze ans plus tard, en avril 2002, Jasper devint une municipalité de l'Alberta après avoir été gérée pendant plusieurs décennies par le Service Canadien des Parcs ${ }^{4}$.

Devenues des municipalités à part entière, ces deux villes se distinguent des autres collectivités (communities). Officiellement, celles-ci sont considérées comme des centres d'accueil pour les visiteurs, mais les situations sont très différentes. À l'exception de Lake Louise Village, Waterton Village et Field dans les parcs des Montagnes, Waskesiu et Wasagaming dans les Parcs des Prairies ont en commun un très faible nombre de résidents mais une très forte présence saisonnière. Ces collectivités sont de dimension réduite (tableau 1) et elles possèdent parfois - ou possédaient à l'époque où le nombre de résidents était plus élevé - une église et/ou une école primaire (Waterton, Field). En revanche, le «village » Lake Louise (PN Banff), construit en 1972, est le produit d'un développement planifié sous l'impulsion de Parcs Canada et d’opérateurs privés en réponse à l'augmentation de la demande touristique. Lake Louise doit rester un centre d'activité du parc regroupant quelques commerces, des établissements d’hébergement (hôtels, motels, lodges, B\&B,

\footnotetext{
3. Depuis l'entrée en application de la Loi sur les Parcs Nationaux (1988), la question de l'intégrité écologique est devenue fondamentale dans la politique de Parcs Canada. Officiellement, le maintien de l'intégrité écologique est défini comme le fait de « gérer les écosystèmes de telle façon à assurer la perpétuité de la diversité génétique, des espèces et des écosystèmes » (Patrimoine Canadien, 1994).

4. Précisons qu’une administration fédérale n’a pas le droit de diriger une Municipalité.
} 
mais ni école, ni église) et les bâtiments de services du parc (centre d'accueil, bâtiments des opérations de Parcs Canada).

\section{UN DÉVELOPPEMENT ASSOCIÉ À LA CROISSANCE DU TOURISME ET DES ACTIVITÉS RÉCRÉATIVES}

En 2005, seuls 6 des 41 parcs nationaux canadiens actuels disposent de ces centres d'accueils. Le tableau 1 montre qu'ils ont été créés dès l'origine des parcs (Banff, Field) lorsqu'ils ne leur préexistaient pas (Waskesiu et surtout Wasagaming). À l'origine, désignés sous le terme de Forest Reserve, ces territoires en réserve étaient conçus comme des espaces dont la fonction consistait à participer au développement économique régional et national (Foster, 1998; McNamee, 1993 ; Héritier, 2002). Certains parcs furent alors dotés de collectivités destinées à fournir les services de base aux visiteurs (hébergement, restauration) à partir des gares situées sur place (Field, Banff, Jasper). Celles-ci furent souvent installées près des lacs présentant un intérêt paysager considérable et permettant de pratiquer la pêche dans un espace naturel (Waterton, Wasagaming, Waskesiu et dans un contexte différent, Lake Louise) (Lothian, 1977; Waiser, 1989).

Pendant plus d'un siècle, l'évolution démographique des collectivités a été marquée par deux tendances très nettes : la première est celle d'une croissance forte pour Banff et Jasper et la seconde traduit un développement faible suivi d'une réduction régulière des résidents permanents pour les centres d'accueil. La tendance principale est illustrée par les villes actuelles de Banff, de Jasper et par le centre d'accueil Lake Louise Village. À partir de sa fondation en 1886, Banff - alors une simple collectivité identique à la plupart des villes pionnières américaines - a gagné rapidement des résidents : comptant 211 habitants en 1901, elle atteint 3429 habitants en $1961^{5}$. En 2001, la population atteint 7125 résidents permanents et un recensement municipal de 2005 proposait une estimation de plus de 8000 résidents. À Jasper, le développement a été plus lent. La fonction ferroviaire fut complétée par la fonction d'accueil. La croissance générale fut plus modérée et moins rapide que celle de Banff pour atteindre 3422 résidents au recensement de 1981, 4301 habitants en 1996, 4700 en 2004. Développé à partir de la fin des années 1950, le centre d'accueil Lake Louise Village regroupe plus de 1900 résidents en 2005 (Sanguin et Gill, 1991 ; Héritier, 2002 ; Parks Canada, 2005).

Les collectivités des autres parcs ont connu un développement moins important et le nombre de résidents n'a cessé de décroître à Field, à Waterton, à Waskesiu et à Wasagaming depuis les années 1960. Cette tendance s'explique de plusieurs manières : l'évolution démographique tout d'abord. Les responsables des parcs ${ }^{6}$ rappellent que les chalets appartiennent souvent de longue date - parfois depuis l'origine - à la même famille. Les propriétés ont, jusqu'à présent, été transmises de génération en génération, révélant un fort attachement aux lieux et aux parcs. Toutefois, les générations de jeunes propriétaires ne travaillent plus et ne résident plus dans les parcs. Leurs maisons deviennent des résidences secondaires et la population permanente diminue régulièrement. La deuxième explication est liée à l'augmentation de la valeur des biens immobiliers qui rend attractive la revente des biens familiaux, qu'il s'agisse des chalets ou des cabins ${ }^{7}$. À Wasagaming, par exemple, on observe depuis quelques années à une mutation de la propriété foncière ${ }^{8}$ : les descendants des premiers possesseurs de logements revendent à d'autres particuliers leurs biens en bénéficiant d'une belle plus-value. Enfin, la politique officielle de Parcs Canada est fortement marquée par la question de l'intégrité écologique et l'Agence fédérale est favorable à la réduction de l'occupation permanente. Comme le précise la Loi sur l'Agence Parcs Canada de 1998, l'ob-

5. Les modalités du développement de Banff Townsite sont rappelées dans un article de G. Richez publié en 1991 (cf. bibliographie).

6. Les entretiens avec les Directeurs du PN Lacs Waterton et du PN Riding Mountain allaient dans le même sens.

7. Entretien effectué en 2005 avec le Directeur et le Townsite Manager du Parc National Riding Mountain.

8. Le nombre de vente pendant la période 2000-2005 a été plus important que dans les vingt dernières années et la valeur des biens a plus que doublé pendant cette période (Entretien avec les responsables du parc). 


\begin{tabular}{|c|c|c|c|c|c|c|c|}
\hline $\begin{array}{l}\text { Nom de la } \\
\text { collectivité }\end{array}$ & Banff & $\begin{array}{c}\text { Lake Louise } \\
\text { Village }\end{array}$ & Field & $\begin{array}{l}\text { Waterton } \\
\text { Village }\end{array}$ & Jasper & Wasagaming & Waskesiu \\
\hline $\begin{array}{l}\text { Fondation (date } \\
\text { de l'arpentage) }\end{array}$ & 1886 & $\begin{array}{c}1972 \\
\text { (forme actuelle) }\end{array}$ & 1904 & 1910 & 1913 & 1916 & 1925 \\
\hline $\begin{array}{l}\text { Parc national } \\
\text { du Canada }\end{array}$ & Banff & Banff & Yoho & Lacs Waterton & Jasper & Riding Mountain & Prince Albert \\
\hline $\begin{array}{l}\text { Création du Parc } \\
\text { (ou de la réserve) }\end{array}$ & 1885 & - & $1884 / 1886$ & 1895 & 1907 & $1929-1930$ & 1927 \\
\hline $\begin{array}{l}\text { Superficie Parc } \\
\text { National (km2) }\end{array}$ & 6641 & - & 1913,10 & 525 & 10878 & 2976 & 3875 \\
\hline $\begin{array}{l}\text { Visiteurs des parcs } \\
\text { (moyenne par an } \\
\text { pour 2000-2005) }\end{array}$ & 3084643 & - & 562298 & 391716 & 1829547 & 259360 & 227987 \\
\hline Statut & $\begin{array}{c}\text { Municipalité } \\
\text { "incorporée" (1990) }\end{array}$ & collectivité & collectivité & collectivité & $\begin{array}{c}\text { Municipalité } \\
\text { "incorporée" (2002) }\end{array}$ & collectivité & collectivité \\
\hline $\begin{array}{l}\text { Superficie de la } \\
\text { collectivité }\end{array}$ & $4,85 \mathrm{~km} 2$ & $1,31 \mathrm{~km} 2$ & $0,38 \mathrm{~km} 2$ & 85 ha & $3,15 \mathrm{~km} 2$ & 179 hectares & $5,3 \mathrm{~km} 2$ (530 ha) \\
\hline $\begin{array}{l}\text { Origine du } \\
\text { toponyme }\end{array}$ & $\begin{array}{l}\text { Toponyme } \\
\text { écossais }\end{array}$ & Lac (Louise) & $\begin{array}{c}\text { De Cyrus West Field, } \\
\text { parrain du premier câble } \\
\text { transatlantique (**) }\end{array}$ & $\begin{array}{c}\text { Officier } \\
\text { britannique }\end{array}$ & $\begin{array}{l}\text { Nom d'un } \\
\text { trappeur }\end{array}$ & $\begin{array}{l}\text { Mot Cree pour } \\
\text { "Eau Claire" }(* *)\end{array}$ & $\begin{array}{l}\text { Mot Cree pour The } \\
\text { place where Red Deer } \\
\text { are Plentiful (\#) }\end{array}$ \\
\hline $\begin{array}{l}\text { Effectif de } \\
\text { rẼsidents (1) }\end{array}$ & $\begin{array}{l}6098(1996) \\
7125(2001)\end{array}$ & $1915(2000)$ & $130(2005)$ & $\begin{array}{l}100(2001) \\
50(2005)\end{array}$ & $\begin{array}{l}4301(1996) \\
4700(2001)\end{array}$ & $\begin{array}{l}55(2001) \\
40(2005)\end{array}$ & $50(2005)$ \\
\hline \begin{tabular}{|c|}
$\begin{array}{c}\text { Part des visiteurs } \\
\text { se rendant dans la } \\
\text { collectivité }\end{array}$ \\
\end{tabular} & $80 \%$ & Plus de $60 \%$ & - & Plus de $80 \%$ & Plus de $60 \%$ & $94 \%$ & $85 \%$ \\
\hline $\begin{array}{c}\text { Nombre de } \\
\text { personnes/jour } \\
\text { (résidents + visiteurs) }\end{array}$ & 30 à 35000 & $\begin{array}{c}\text { Environ } \\
20000\end{array}$ & $\begin{array}{l}\text { Quelques } \\
\text { centaines }\end{array}$ & $\begin{array}{l}\text { Entre } 3000 \\
\text { et } 4000\end{array}$ & $\begin{array}{l}\text { Environ } \\
20000\end{array}$ & $\begin{array}{c}12000 \mathrm{à} \\
15000\end{array}$ & $\begin{array}{l}\text { Environ } \\
9000\end{array}$ \\
\hline \multicolumn{8}{|c|}{$\begin{array}{l}\text { (1) : Concerne les résidents permanents seulement. } \\
\text { Les résidents temporaires ne sont pas comptabilisés. }\end{array}$} \\
\hline
\end{tabular}

Tableau 1 : Situation générale des collectivités Townsites and Communities within Canadian Western Parks

jectif est de maintenir ou de restaurer l'intégrité écologique des parcs nationaux [...] de subordonner l'usage des parcs nationaux [...] à leur intégrité écologique [...] de gérer l'utilisation par les visiteurs et les touristes des parcs nationaux (Agence Parcs Canada, 2000).

La politique officielle est aujourd'hui restrictive, ou pour le moins limitative en matière d'usage permanent (Richez, 2000a). Pourtant, pendant plusieurs décennies, ces collectivités se sont développées pour répondre aux besoins du personnel des parcs, des entrepreneurs locaux et des employés, des résidents et des visiteurs.

\section{UN DÉVELOPPEMENT BÉNÉFICIANT DE SITUATIONS RÉGIONALES PRIVILÉGIÉES}

L'histoire du développement de ces collectivités est assez bien documentée et les nombreuses études permettent se forger une idée précise des modalités du développement de ces sites urbains.

Dans la plupart des cas, le chemin de fer a permis de créer les conditions d'un tourisme élitiste, au moins pendant les premières décennies. L'accès ferroviaire permettait de proposer un dépaysement aux populations urbaines de l'Est du pays, et au-delà des voyageurs internationaux. Déplacement transcontinental et voyages internationaux (américains et européens, notamment des anglais) assuraient l'essentiel du développement touristique, réclamant des équipements adaptés à une clientèle argentée. Pour répondre à cette recherche de clientèle du chemin de fer, Van Horne, alors président, décida de construire de grands hôtels qui contribuèrent au développement de centres d'accueil pour les visiteurs.

Toutefois, la croissance fut rapidement assurée grâce aux visiteurs originaires des provinces où les parcs avaient été créés et des provinces voisines. De véritables bassins touristiques, dépassant parfois l'échelle provinciale, se constituèrent dans l'environnement des parcs. Dans certains cas, une ville importante contribuait à stimuler la croissance des centres d'accueil : pour Banff par 
exemple, la facilité d'accès et la proximité de Calgary ont contribué à accroître le développement de la ville (Scace, 1969, 1979). Au Manitoba, la population de villes importantes telles que Winnipeg, à la recherche de sites et d'espaces récréatifs, avaient contribué à établir un secteur de chalets sur la rive sud du lac Clear. Le site servit de base au développement des activités récréatives et touristiques après la création du Parc National Riding Mountain et a été baptisé Wasagaming en 1930 (Lothian, 1977; Tabulenas, 1983). Dans le cas de Waskesiu, enfin, le parc est considéré comme un véritable «terrain de jeu " par les habitants de la province (Waiser, 1989) et le centre d'accueil est parfois assimilé au parc dans son entier. On peut en effet lire dans le plan directeur du PN Prince Albert que «for many visitors, the community of Waskesiu is Prince Albert National Park » (Environment Canada, 1988).

Finalement, les réserves forestières, devenues parcs nationaux, ont changé de fonction. Elles sont devenues des espaces récréatifs dans lesquels des visiteurs locaux, régionaux, provinciaux, nationaux et internationaux peuvent goûter les joies de la wilderness. Face à l'augmentation de la demande des visiteurs, les parcs se sont dotés d'infrastructures et de centres d'accueil qui ont évolué pour devenir des collectivités et des villes à part entière, laissant entrevoir les difficultés liées à la gestion d'espaces dotés de fonctions urbaines à l'intérieur de parcs nationaux.

\section{Problèmes de types urbains et difficultés de gestion}

Avec l'augmentation considérable de la fréquentation dans les parcs nationaux canadiens depuis les années 1960, les centres d'accueil font l'objet d'une augmentation saisonnière considérable du nombre de visiteurs et de ses corollaires : demande en eau, production d'eaux usées et de déchets solides, augmentation de la circulation, etc. Ces évolutions et la croissance démographique dans certaines collectivités ont imposé de nouvelles conditions d'exercice de l'autorité urbaine.

\section{LES PROBLÈMES LIÉS AU NOMBRE DE VISITEURS}

Plusieurs centaines de milliers de visiteurs se rendent chaque année dans les parcs auxquels cette étude est consacrée (tableau 1 et fig. 2). Pour la période 2000-2005, le nombre moyen de visiteurs varie entre 228000 personnes environ dans le PN Prince Albert et plus de trois millions d'individus dans le PN Banff. Les villes et les collectivités, faisant office de centre d'accueil et de lieu de ravitaillement (carburant, alimentation, etc.), accueillent en général entre $70 \%$ et $95 \%$ des visiteurs des parcs. La synchronie de la fréquentation des parcs et du développement des communautés n'a alors rien de surprenant.

La figure 2 montre la situation contemporaine des sites urbains. À la différence d'effectifs en matière de résidents, il faut ajouter une fréquentation très irrégulière, marquée par un rythme où alternent des phases de très basse fréquentation opposées à des périodes de surpopulation relative. Cette figure laisse aisément imaginer les problèmes qui apparaissent alors en terme d'infrastructures et de gestion des flux et reflux de visiteurs. Cette situation était évoquée récemment par une journaliste banffite : elle rappelait que « la ville [qui dépasse les 8000 résidents] avait à prendre en charge les frais d'entretien des services essentiels pour une moyenne de 17000 personnes et avait dû construire une station d'épuration des eaux usées d'une capacité de plus de 30000 personnes » (Svatek, 2004). Ce commentaire soulève en réalité une question bien plus polémique, à savoir le surcoût, pour le budget de la ville de Banff - avec ses implications fiscales - induit par la prise en charge traitement des eaux.

Avant 1990, la gestion des collectivités était intégralement à la charge de Parcs Canada, qui pouvait occasionnellement organiser des consultations publiques. Depuis cette date, les compétences ont évolué parallèlement au partage de l'autorité urbaine. 


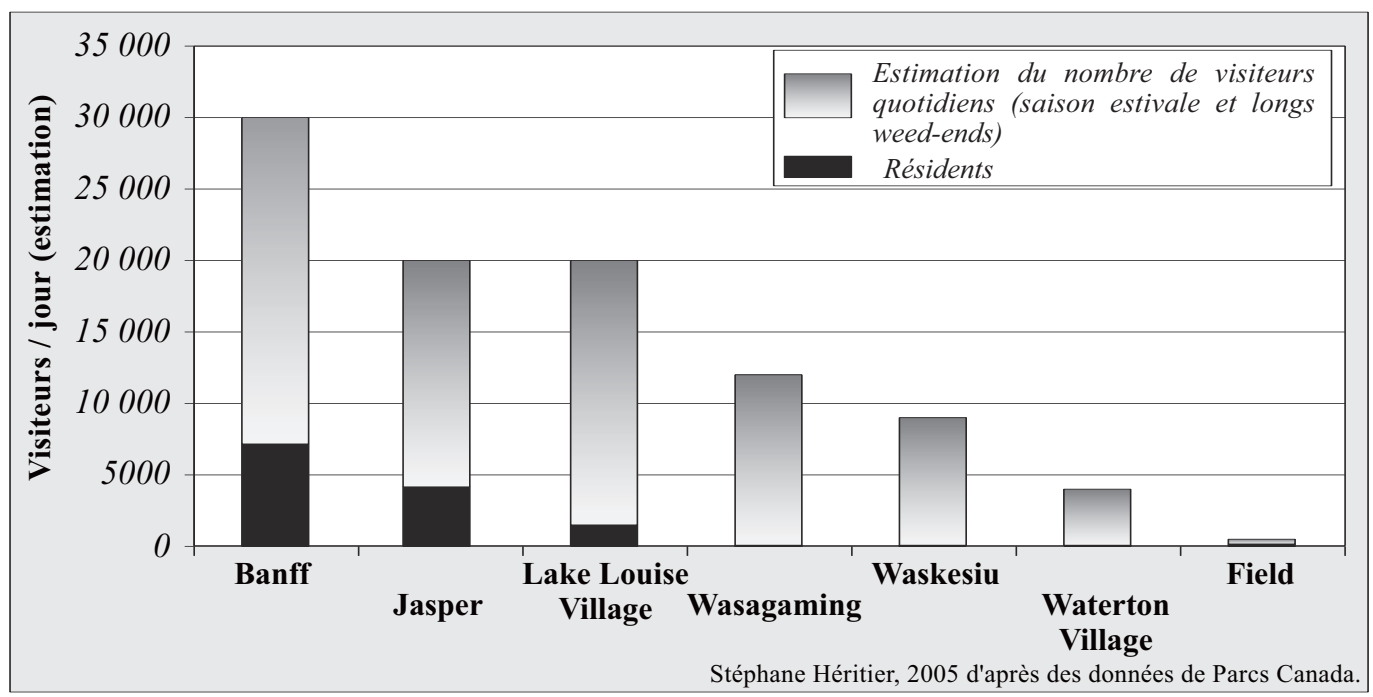

Figure 2 : Estimation de la fréquentation moyenne quotidienne des villes et collectivités des parcs nationaux Estimated daily visitation (average) for townsites and communities

\section{COMPÉTENCES ET AUTORITÉ URBAINE : VILLES ET COLLECTIVITÉS}

Au Canada, les territoires situés à l'intérieur d'un Parc National ${ }^{9}$ sont intégralement la propriété de l'État fédéral ${ }^{10}$. Ailleurs dans le monde, les situations diffèrent : en Europe, les situations sont extrêmement variées, l'État ayant une position quasiment exclusive (Finlande, Suède, Norvège par exemple) ou partageant la propriété foncière avec d'autres acteurs (Pays-Bas, Pologne, Italie) (Richez, 1992); en France, la propriété des terres en Zone Centrale des parcs nationaux est répartie entre différents acteurs : État, communes, particuliers (Atlas du Parc National des Écrins, 2000 ; Laslaz, 2004).

L'année 1990 ouvrit une nouvelle ère en terme de gestion des collectivités à l'intérieur des parcs parce qu'elle se traduisit par un mouvement de reclassement de certaines collectivités. Celui-ci consista en la modification du statut de certains espaces urbanisés dans les parcs. En effet, en 1990 puis en 2002, les municipalités de la ville de Banff et de Jasper remplacèrent respectivement les collectivités autrefois gérées intégralement par Parcs Canada. Ces deux villes (par les services urbains qu'elles proposent et par leurs effectifs démographiques) constituent des cas exceptionnels au sein du système canadien des parcs.

Ainsi, Banff puis Jasper sont devenues des municipalités dites « incorporées » (incorporated), c'està-dire intégrées au système légal de la Province d'Alberta, devenant ainsi des entités urbaines au sein des parcs. Les modifications majeures entérinées par les ententes juridiques définissent les compétences des nouvelles municipalités et leurs obligations au regard de la législation sur les

9. Cette remarque ne tient pas compte de la nouvelle dénomination des anciens Parcs Provinciaux du Québec devenus Parcs Nationaux du Québec.

10. Rappelons un élément important de la constitution des parcs nationaux canadiens : En 1885, lorsque la « Réserve des Sources Thermales Banff »-Banff Hot Spring Reservation est créée, l'essentiel des Territoires de l'Ouest et du Nord (correspondant globalement aux provinces actuelles des Prairies et aux Territoires du Nord) sont la propriété de la Couronne (l'État). Ces terres, avaient été rachetées à la Compagnie de la Baie d'Hudson peu après la création de la Confédération canadienne et l'État en était propriétaire. En 1930, l'État fédéral confia la propriété de ces terres aux Provinces à l'exception des Territoires du Nord qui restèrent sous contrôle fédéral. Depuis cette date, lorsque le gouvernement prévoit de créer un Parc National dans une province, il lui faut acheter les terres aux différents propriétaires fonciers (Provinces ou particuliers). 
Parcs Nationaux. Rappelons que dans tous les cas, la législation fédérale est applicable dans la mesure où les deux municipalités louent finalement des terres qui demeurent la propriété de l'État. De fait, les villes doivent respecter les cadres législatifs essentiels tels que la Loi sur les parcs nationaux - National Park Act de 1998 et ses évolutions incluses dans la Loi sur les Parcs Nationaux du Canada - Canada National Park Act ${ }^{11}$ de 2000 ainsi que la loi canadienne de 1992 sur l'évaluation environnementale.

Depuis leur «incorporation », les municipalités se trouvent dans la même situation que n’importe quel détenteur d'un bail commercial ou résidentiel. Elles doivent en conséquence s'acquitter d'une rente annuelle, sorte de droit d'usage, fixée contractuellement de manière forfaitaire au moment de l'entente entre le gouvernement fédéral et celui de l'Alberta ${ }^{12}$ (Town of Banff Incorporation Agreement, 1989; Municipality of Jasper, 2002). Fait original, la ville est littéralement locataire de son espace support. Les municipalités doivent également prendre en charge les tâches qui incombaient autrefois au Service Canadien des Parcs. Les conditions d'exercice de l'autorité urbaine sont sérieusement règlementées. Si Banff et Jasper disposent de l'indépendance urbaine, celle-ci demeure encadrée par les réglementations nationales qui sont en vigueur dans les parcs nationaux. Dans les deux cas, le gouvernement municipal est chargé de l'entretien et de la réfection des routes et des trottoirs, de la collecte des poubelles, de l'adduction et du traitement des eaux, de la protection contre les incendies et des services communautaires (Town of Banff, 1998; Parks Canada, 2005). Toutefois, à la différence de Jasper, la municipalité de Banff dispose de l'autorité en terme de planification et de gestion urbaine tandis qu'à Jasper, le gouvernement fédéral, par l'intermédiaire de Parcs Canada, conserve toute autorité sur la planification urbaine, la destination de l'usage foncier, le développement et les questions environnementales.

À la différence de ces deux villes, les cinq autres collectivités demeurent sous la tutelle pleine et entière de Parcs Canada, qui est chargé de les gérer en assurant le respect de la législation en matière de protection de l'environnement. Dans une certaine mesure, la gestion municipale dédouane en partie Parcs Canada des orientations de gestion, mais, bien souvent, l'agence fédérale est sous le feu des critiques. Pourtant, il faut rendre justice aux représentants de cette institution - directeurs des parcs et Townsite Manager - qui cherchent la plupart du temps à faire participer les acteurs locaux aux décisions. Toutefois, les crispations demeurent souvent fortes tant les intérêts semblent contradictoires.

\section{La recherche de l'équilibre entre la gestion urbano-touristique et la protection du territoire}

Les trois dernières décennies ont été celles d'une contestation et d'une critique parfois systématique du Dual Mandate que le Service Canadien des Parcs devait mettre en œuvre : mandat dual, que l'on pourrait résumer par l'obligation qui lui était faite de mettre tout en œuvre pour protéger des espaces de nature sauvage tout en créant les conditions favorables pour les rendre accessibles à un large public. Ce paradoxe, commun à la majorité des parcs nationaux, a pendant longtemps été le fondement de la critique de l'action de Parcs Canada, tant de la part du secteur privé Parcs Canada était considéré comme trop protectionniste - que de la part des défenseurs de l'environnement, contestant les actions en faveur de l'ouverture au public.

La responsabilité de Parcs Canada et des deux municipalités est désormais une responsabilité complexe, dans la mesure où la protection des biens privés et publics, la protection des personnes et la protection de l'environnement (maintient et restauration de l'intégrité écologique notam-

11. Elle s’intéresse notamment aux collectivités de parcs et prévoit des « mesures de contrôle du développement commercial dans les sept collectivités de parcs [...], d'assurer un équilibre entre les intérêts des résidents locaux en matière de croissance et de développement de leur collectivité, et l'intérêt national en faveur du maintien de l'intégrité écologique des Parcs » (Loi sur les Parcs Nationaux du Canada, 2000, Chapitre 32). Elle insiste sur la nécessité que chaque collectivité dispose d'un plan, sur le fait que les limites de toutes les collectivités, le zonage commercial et les superficies commerciales utilisables sont approuvés par une loi du Parlement et ne peuvent être modifiés sans un débat préalable et une décision du parlement du Canada.

12. La valeur fut fixée à hauteur de 550000 dollars canadiens pour la ville de Banff et 475000 dollars pour la municipalité de Jasper. 
STÉPHANE HÉRITIER

ment) constituent les cadres généraux de l'intervention. La manière dont a été abordée la gestion des risques d'incendie pour les collectivités illustre le difficile équilibre à trouver, comme le révèle également le bilan inégal des quinze années de gestion municipale de Banff. Partout pourtant, les enjeux demeurent importants.

\section{LA GESTION DES RISQUES D'INCENDIE, AUX LIMITES \\ ENTRE LA PROTECTION DE L'ENVIRONNEMENT \\ ET LA PROTECTION DES AMÉNAGEMENTS URBAINS}

Parmi les nombreux enjeux au cœur de la gestion des sites urbains dans les parcs nationaux, la prévention et le contrôle des incendies de forêts constitue l'un des aspects remarquables. Rappelons qu'en 1988, le Parc National Yellowstone, aux États-Unis, fut marqué par de gigantesques incendies affectant $36 \%$ de la superficie du parc (National Park Service, 2002). Cet événement accéléra la prise de conscience de la probabilité d'occurrence de dommages pour les aménagements situés au cœur des parcs canadiens : auberges, centres d'accueil, villes.

À partir de cette date, Parcs Canada, déjà sensibilisé à ces questions, en fit l'une de ses préoccupations majeure en raison du nombre de sites sensibles (logements commerciaux privés, villes ou collectivités). Deux raisons peuvent être invoquées : la première est liée à l'évolution de la prise en charge des incendies dans les parcs nationaux canadiens. Au début, les parcs constituaient des réserves forestières et ils étaient logiquement combattus. Des photographies prises entre les années 1885 et 1930 montrent que les forêts de la vallée de la Bow autour de Banff et de la vallée Athabasca à l'aval de Jasper, présentaient une végétation ligneuse très dispersée, héritage des incendies effectués par des causes naturelles (éclairs) et ou par les activités anthropiques (chemin de fer, mines, etc. - White, 1985). La politique en faveur de la reconstitution des forêts constitua un objectif majeur.

Progressivement, la végétation a repoussé, protégée par une activité forestière aujourd'hui interdite. En conséquence, la couverture ligneuse a actuellement atteint un stade de maturité avancé dans la quasi-totalité des parcs (couvert dense, réservoir de bois mort très important dans la majeure partie des régions concernées). Dans les années 1990, toutes les communautés étaient serties d'un écrin forestier, qui concordait avec l'image d'îlots de civilisations au milieu de la wilderness, mais tous les responsables des parcs s'accordaient à dire qu'il s'agissait de « bombes à retardement »- les incendies de 2003 dans les Parcs voisins de Banff, Kootenay et Yoho, l'ont bien montré. Si les feux de forêts étaient considérés comme des processus naturels de régénération de la végétation, les incendies n'en constituaient pas moins un danger considérable pour les personnes, les biens et la valeur économique de ces sites.

La situation a évolué pour s'orienter vers une politique mixte fondée sur le «laisser faire » - l'incendie considéré comme processus naturel - et la prévention. Dans la plupart des parcs nationaux canadiens, et a fortiori dans ceux disposant de centres d'accueil permanents, une politique en faveur de feux dirigés (prescribed burns) a été mise en place. Ces secteurs incendiés - réduisant ainsi le combustible disponible - ont pour fonction de servir de pare-feu en cas d'incendie majeur et de réduire les risques d'incendies pour les collectivités dont plus de $90 \%$ du bâti utilise le bois comme matériau de construction (Waterton, Field notamment). Le bâti est également dense (Wasagaming) et souvent intégré aux espaces forestiers, couvert boisé parfois continu jusqu'à l'intérieur des limites des communautés de Banff et Waskesiu. La mesure la plus récente réside dans la création d'une zone tampon établie directement autour des sites urbains.

À Waskesiu par exemple, l'administration du parc a décidé d'effectuer des opérations de protection préventive de la collectivité. La photo 1 montre qu'un périmètre a été partiellement déboisé, notamment à proximité des bâtiments, tandis qu'une bande intermédiaire a été éclaircie et nettoyée (enlèvement du bois mort, élimination des branches inférieures) afin d'arrêter la progression d'un incendie potentiel (cf. Plans communautaires; Weir et Pidwerbeski, 2000 ; Durnin, 2002). 


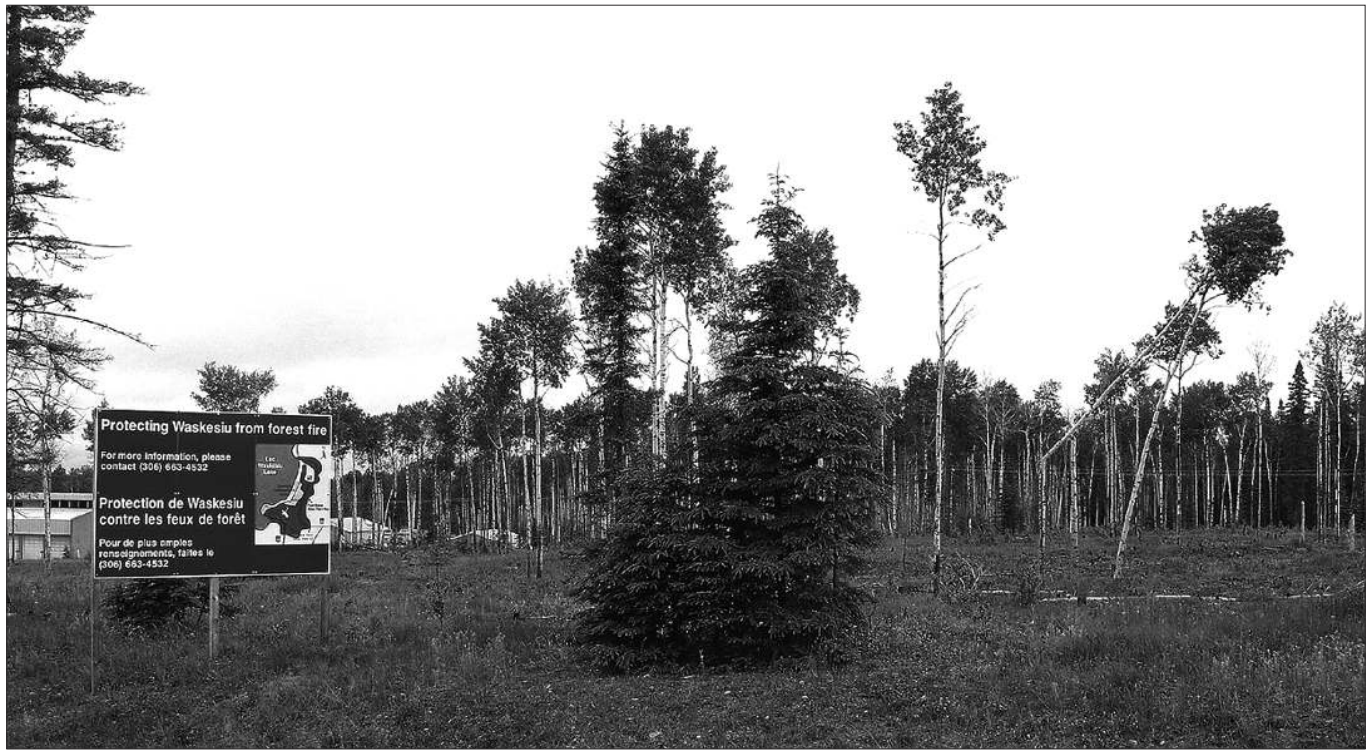

Photo 1 : Aménagement d'un périmètre de protection contre les incendies à Waskesiu (PN Prince Albert, Saskatchewan) (cl. S. Héritier, 2005)

Waskesiu Community Fire Break (Prince Albert National Park)

Le cas de Waskesiu est caractéristique de ce qui a été effectué ou de ce qui est en discussion actuellement pour les autres collectivités. Toutefois, seules ces mesures se révèlent actuellement efficaces pour protéger des biens privés et collectifs mais elles provoquent parfois des grincements de dents, en raison des modifications esthétiques qu'elles supposent.

Dans le cas de l'ensemble des collectivités, Parcs Canada a réussi à établir des règles en matière d'aménagement et de prise en charge des problèmes urbains - à grands renforts d'investissements très coûteux parfois. La ville de Banff s'est distinguée de ce mode de gestion spécifique aux parcs depuis suffisamment longtemps pour qu'il soit intéressant de proposer un bilan d'une action municipale autonome au cœur d'un parc national.

\section{LA VILLE DE BANFF : TENTATIVE DE BILAN APRÈS 15 ANS DE GESTION MUNICIPALE}

En 1991 l'année qui suivait «l'incorporation » de Banff, G. Richez concluait son article (Richez, 1991) par des interrogations qui méritent de nourrir le présent travail : l'auteur s’interrogeait sur « les possibilités réelles de contrôle et de maîtrise des incidences de la ville sur son environnement immédiat », les risques de " "surexploitation" des richesses » par le secteur touristique et sur la pertinence de compléter le dispositif réglementaire de manière adéquate afin "d'éviter tout dérapage et en cas de besoin, de contenir les excès »(Richez, 1991).

En 1994, Lowry écrivait à son tour que l'autonomie municipale inquiétait certaines personnes parmi les employés de Parcs Canada et parmi une partie de la population plus favorable à la protection de l'environnement qu'au développement commercial, sonorité dissonante par rapport aux propos tenus par le directeur du parc qui insistait plutôt sur l'établissement de relations de confiance entre la ville, le secteur privé et l'administration fédérale (Lowry, 1994).

Quel est l'état actuel de la relation entre les différents partenaires en 2005 ? Depuis 1990, les relations ont évolué en fonction des orientations gouvernementales. D’une manière générale, le secteur privé ne parle pas d'une voix unique et deux tendances peuvent être distinguées : la tendance « dure » qui souhaite et clame un développement plus important, une progression de la croissance et des aménagements touristiques; la tendance «souple », composée d'entrepreneurs 
privés qui ont intégré les modes de fonctionnement et de développement économique en relation étroite - et en phase - avec le parc (en tant qu'espace et en tant qu'administration). Ceux-ci sont en général en accord avec la politique mise en place par Parcs Canada et tentent de moduler leur action en fonction des évolutions législatives, parfois perçues comme contraignantes même lorsqu'elles sont reconnues comme nécessaires.

Entre 1990 et 2005, les deux périodes les plus tendues ont été les années 1991-1992 et les années 1995-1998, marquées par la préparation d'un plan d'aménagement municipal (publié en 1992), d'un plan communautaire pour la ville de Banff (1997) et par la révision du plan directeur du Parc National Banff incluant les limitations de la croissance de la ville (Town of Banff, 1998; Parks Canada, 2004). En 1997, le projet de développement initial proposé par la ville (avec ses volets démographiques, commerciaux et immobiliers) avait été préalablement approuvé par la direction du Parc National Banff, dans la mesure où le projet respectait la législation en vigueur. Toutefois, la ministre du Patrimoine de l'époque Sheila Copps, exigea une révision à la baisse du projet de développement commercial et imposa une législation très restrictive à Banff, en partie sous la pression des lobbies environnementalistes ${ }^{13}$.

Depuis lors, en dépit des tensions qui surviennent parfois, dialogue et confiance mutuelle semblent de rigueur entre la municipalité, Parcs Canada, et la majeure partie du secteur privé qui doit se plier aux règles générales sur les parcs nationaux - ce qui n’exclue pas un lobbying actif auprès de la municipalité. L'exemple le plus récent est lié à l'attribution des dernières superficies commerciales à Banff. L'amendement 2004 du plan de gestion du Parc National Banff fixait les limites strictes du développement commercial autorisé à 350000 pieds carrés. Face à cette pénurie foncière, les surfaces commerciales furent attribuées par tirage au sort, effectué en 2005 à partir de projets présentés par des entreprises privées satisfaisant aux réglementations en vigueur dans les parcs. Les acteurs privés ayant bénéficié des surfaces aménageables doivent réaliser leurs projets dans les cinq années sous peine de voir leur attribution suspendue et être remise « en jeu » (Taylor et Ryall, 2003; Parks Canada, 2004; Winks, 2005 et entretiens).

La figure 3 tente de synthétiser les relations entre les différents acteurs qui interviennent dans la prise de décision à Banff. Si les partenaires et les observateurs reconnaissent que les relations s'améliorent et que la confiance entre les partenaires existe, ces relations demeurent le fruit d'un équilibre précaire. La figure permet de percevoir qu'il existe plusieurs groupes aux intérêts ou aux conceptions divergentes concernant le développement de la ville et sa place au cœur d'un parc national. Si Parcs Canada a laissé la gestion de la ville à la municipalité, l’Agence conserve un contrôle incontestable de la situation.

Les partenaires privés, en revanche, ont pris plus de poids, mais le contrôle exercé par l'administration fédérale demeure un puissant garant d'une gestion prudente : par exemple, Parcs Canada a limité les activités et imposé un moratoire sur les constructions dans des secteurs de la ville servant de corridors fauniques, et la croissance de la ville est soumise à une réglementation draconienne. Les partenaires privés ont, dans leur grande majorité, accepté que le développement dans la ville de Banff devait intégrer la particularité et la situation de la ville, dans un souci de pérennité, sinon de durabilité. La législation canadienne a, en la matière, imposé une remise en cause des modalités du développement économique, d'autant plus facile à effectuer que la fréquentation a connu un affaissement depuis 2001 .

\section{Conclusion - Les réussites d'une gestion concertée}

Hérités des premières décennies d'existence des parcs, les centres d'accueil ont toujours constitué des foyers de peuplement et d'activité majeurs pour ceux-ci. Leur évolution fut fonction de l'usage diversifié des ressources touristiques. Toutefois, l'évolution récente suit deux orientations distinctes : la reconnaissance municipale pour les collectivités qui ont atteint des effectifs démo- 


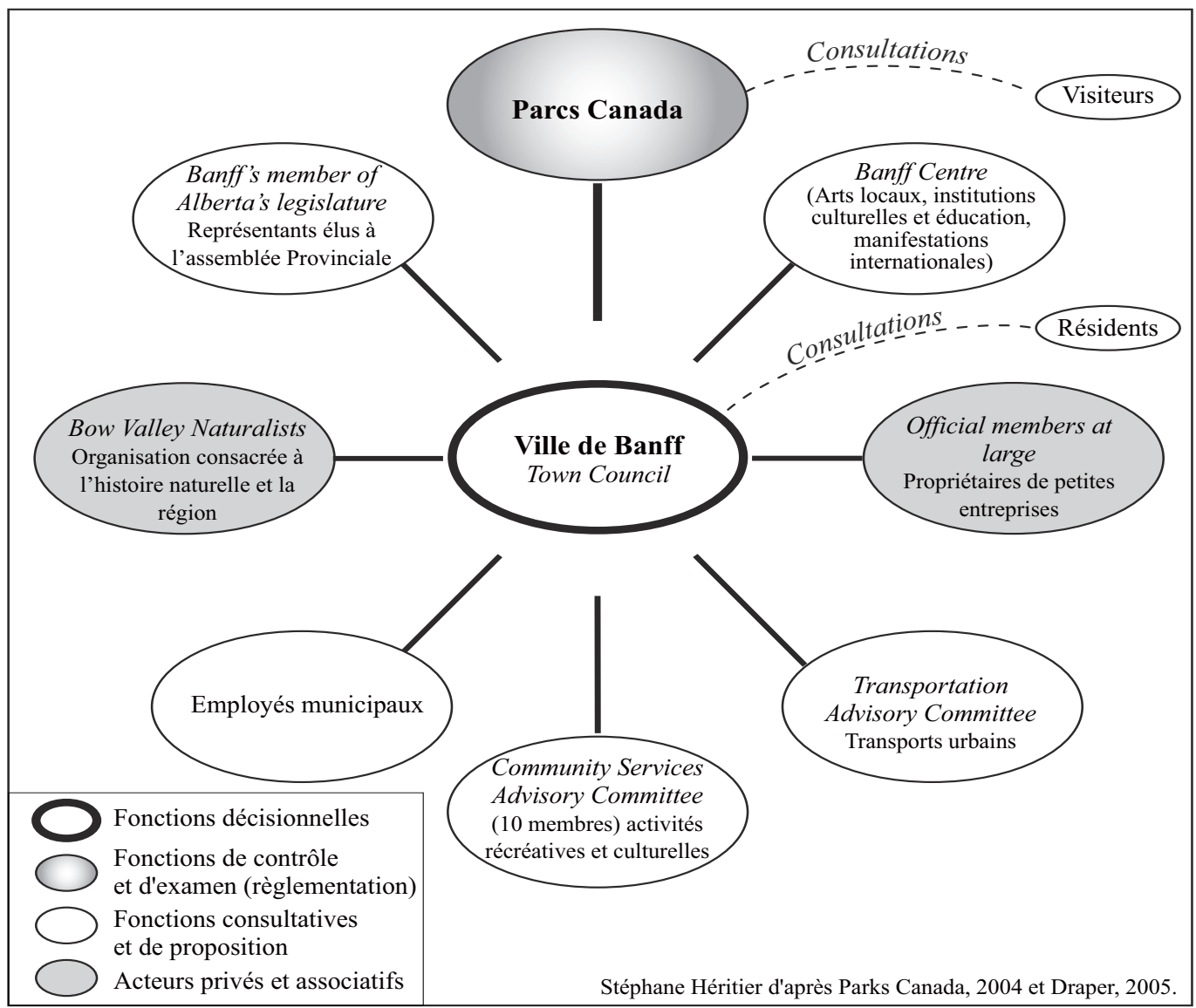

Figure 3 : Complexité de la prise de décision à la municipalité de Banff Town of Banf: Complexity in Decision Making

graphiques et des fonctions urbaines à Banff et Jasper, le déclin progressif de l'occupation permanente au profit d'un usage ponctuel ou saisonnier (Waterton, Waskesiu, Wasagaming, Field), le maintien de collectivités aux strictes fonctions d'accueil (Lake Louise).

La gestion des sites urbains est dans tous les cas marquée par un décisionnaire principal, qu'il s'agisse de la municipalité ou de Parcs Canada. A contrario des municipalités de l'Alberta, celles de Banff et Jasper doivent compter avec un interlocuteur décisif, qui encadre et parfois limite leur champ d'action. Toutefois, on retrouve partout la même volonté de concertation et de cogestion, qui se traduit par la consultation fréquente des acteurs locaux, des résidents et des visiteurs. Le Parc National Riding Mountain présente une situation exemplaire en la matière dans la mesure où l'élaboration du Plan communautaire de Wasagaming a donné lieu à la mise en place d'une table ronde regroupant tous les acteurs locaux (décideurs et acteurs ordinaires) qui ont été régulièrement réunis entre 1994 et 2000. L'objectif de ces consultations était d'atteindre un consensus sur les actions à mener dans le cadre du plan de gestion de la communauté (Tandukar, 1999). L'objectif avoué consiste à limiter les effets de rejets des mesures proposées. La démarche consiste à expliquer le bien fondé des mesures et d'obtenir une sorte de consensus sur les mesures à prendre, limitant ainsi les protestations et les tentatives de contournement de la législation. Dans les faits, si les accrochages sont parfois vigoureux dans la presse ou pendant les réunions publiques entre les différents intervenants, la méthode fonctionne plutôt efficacement. 
La tâche des gestionnaires des collectivités - Parcs Canada ou municipalités - n'est pas aisée dans la mesure où la valeur attribuée à ces espaces est particulièrement élevée (Héritier, 1999, 2002). Dans une certaine mesure, les sites urbains répondent à un besoin social aussi important que le désir de Nature exprimé par les visiteurs dans les parcs ${ }^{14}$. La conjonction de ces deux éléments et les réglementations inévitables induites par les sociétés humaines cristallise les enjeux à propos d'espaces d'autant plus importants qu'ils sont réduits. En dépit des plans de gestion et des plans d'urbanisme, certaines questions demeurent en suspens dans le calendrier de l'aménagement local : la question de la gestion des eaux usées - et surtout du coût pour la municipalité - demeure un problème majeur et polémique dans toutes les collectivités en raison de l'irrégularité de la fréquentation et des besoins brutaux de traitement (Wasagaming, Waskesiu, Banff).

La planification urbaine est devenue très restrictive, provoquant le mécontentement récurrent de certains entrepreneurs privés (Waskesiu, Banff). Parfois, cette planification est au ban des accusés lorsque les problèmes de voirie deviennent des causes de tensions entre les résidents et les gestionnaires, accusés de délaisser les collectivités (Waterton et Field jusqu'à une période récente). Il faut ajouter à cela la concurrence spatiale entre résidents et visiteurs, entre les humains et la faune, qui produisent respectivement émoi et colère lorsqu'un grizzli est victime de l'activité humaine ou responsable de la mort d'un visiteur. Par ailleurs, la limitation de croissance à l'intérieur des parcs reporte les problèmes à leurs marges, induisant des rythmes de croissance urbaine très élevés aux espaces situés à l'entrée des parcs, comme c'est le cas pour Canmore au sud-est de Banff (Héritier, 2004) et d'Onanole au Sud de Wasagaming.

Au final, le bon gouvernement demeure délicat à réaliser dans les collectivités et les parcs nationaux de l'Ouest du Canada. Depuis quelques années, les efforts de Parcs Canada ont été facilités par une législation en faveur de la protection de l'environnement grâce à l'usage de la notion de maintien et de restauration de l'intégrité écologique que tous les acteurs doivent respecter dans la gestion quotidienne des parcs, des collectivités, des municipalités présentes dans les parcs nationaux canadiens. L'option qui est alors proposée aux acteurs privés comme aux gestionnaires publics est celle d'un développement qualitatif et plus seulement quantitatif, inaugurant par les actes une véritable durabilité et participant d'une réelle gouvernance.

\section{Bibliographie}

Agence Parcs Canada, 2000. - Intacts pour les générations futures? Protection de l'intégrité écologique par les Parcs Nationaux du Canada, vol. II « Une nouvelle orientation pour les parcs nationaux du Canada », Rapport de la Commission sur l'intégrité écologique des Parcs Nationaux du Canada, Ottawa (Ontario), pagination spécifique.

—, 2004. - Modèle de rapport d'examen préalable type pour les projets courants dans les collectivités des parcs nationaux, Ottawa, Parcs Canada, vol. 1, 92 p.

Atlas du Parc national des Écrins, 2000, Gap, Atlas des Parcs nationaux de France, Parc national des Écrins, ministère de l'Aménagement du Territoire et de l'environnement, 64 p.

DraPER (D.), 2005. - « Working relationships among citizens, town council members and municipal administrators: Implementation of the Banff Community Plan (BCP)», Personal communication presented at the Governance and Decision-Making in Mountain Areas, June 4-8, 2005, The Fifth Conference in the Mountain Communities series.

14. Ce désir de Nature est l'une des justifications majeures du succès rencontré par ces parcs. En parallèle à ces espaces urbanisés, les gestionnaires des parcs nationaux imposent des pratiques inverses, visant à réduire et dans certains cas à interdire les infrastructures d'accueil, afin de ne pas altérer - ou de permettre une restauration de - la wilderness. Le secteur supérieur du Columbia Icefield et du glacier Athabasca, au sud du Parc National Jasper (Sanguin et Gill, 1990), le secteur du Lac Audy dans le Parc National Riging Mountain et plus encore celui des schistes argileux de Burgess dans le Parc National Riging Mountain et plus encore celui des schistes argileux de Burgess dans le Parc National Yoho en constituent des exemples éclairants. 
Durnin (J.), 2002. - Prince Albert National Park Fire Control Plan, Waskesiu Lake, Resource Conservation Prairie and Northern Region, Parks Canada, december 2002, 23 p.

Environment Canada, 1988. - Prince Albert National Park Management Plan, Ottawa, Minister of Supply and Services, Parks, 92 p.

Foster (J.), 1998. - Working for Wildlife, The beginning of Preservation in Canada, Toronto: University of Toronto Press, $2^{\mathrm{e}}$ édition, $297 \mathrm{p}$.

GazetTe du Canada, 2004. - Loi sur les parcs Nationaux du Canada. Décret modifiant l'annexe 4 de la Loi sur les parcs nationaux du Canada, 11 mai 2004, (enregistrement DORS/2004-116), Partie II, vol. 138, $n^{\circ} 11$, p. 706-717.

Héritier (S.), 1999. - « La gestion de l'environnement et du patrimoine dans le Parc National des LacsWaterton (Alberta, Rocheuses canadiennes)», Revue de Géographie Alpine, Tome 87, n 4, p. 49-64.

—, 2002. - Environnement et patrimoine, tourisme et aménagement dans les parcs nationaux des montagnes de l'Ouest canadien (Banff, Jasper, Yoho, Kootenay, Revelstoke, Glacier, Lacs Waterton), Doctorat de Géographie, Université de Savoie (Chambéry-Annecy), 390 p.

—, 2004. - « Les dynamiques spatiales dans les aires protégées : l'exemple des parcs nationaux des montagnes de l'Ouest canadien (Alberta, Colombie Britannique)», Mappemonde, n² 2, 2004 [http://mappemonde.mgm.fr/num2/articles/art04202.html].

Laslaz (L.), 2004. - Vanoise: 40 ans de Parc National; bilan et perspectives, Paris, L'Harmattan, 427 p.

Lothian (W.-F.), 1977. - Histoire des parcs nationaux du Canada, Ottawa, Parcs Canada, volume I, 129 p.

—, 1979. - Histoire des parcs nationaux du Canada, Ottawa, Parcs Canada, volume III, 123 p.

LownY (W. R.), 1994. - The Capacity for Wonder: Preserving National Parks, Washington D.C., The Brookings Institution, $280 \mathrm{p}$.

McKim (W.), 1970. - « Townsite Administration and Management in Canadian National Parks », p. 177-186, dans Nelson (J. G.), 1970. - Canadian Parks in Perspective, Montréal, Harvest House Ltd., 343 p.

McNameE (K.), 1993. - « From wild places to endangered spaces: a history of Canada's National Parks », p. 17-44 dans Dearden (P.), Rollins (R.) (dir.). - Parks and protected areas in Canada, plannig and management, Toronto, Oxford University Press, $336 \mathrm{p}$.

Municipality OF JASPeR, 2002. - Policy Manual, including the Agreement for the Establishment of Local Government In the Town of Jasper, Jasper, Alberta, $9 \mathrm{p}$.

National Park SERVICE, 2002, Yellowstone Resources \& Issues. An annual compendium of information about Yellowstone National Park, Division of Interpretation, Yellowstone Natioanl Park, Mammoth Hot Springs, Wyoming, 180 p.

Parcs Canada, 1999. - Plan Communautaire de Field, Parc National Yoho, Parcs Canada, juillet 1999, Ottawa, Parcs Canada, ministère du Patrimoine, 78 p.

—, 2000. - Plan Communautaire de Waskesiu, Ottawa, Ministère du Patrimoine, 47 p.

-, 2000-LW. - Parc national des Lacs Waterton, Plan Communautaire de Waterton, Ottawa, Parcs Canada, Patrimoine canadien, $80 \mathrm{p}$.

—, 2000-J. - Parc national Jasper du Canada, Plan directeur, mai 2000, Ottawa, Patrimoine canadien, Parcs Canada, 78 p.

—, 2001-J. - Plan d'utilisation des terres de la communauté de Jasper, Parc National Jasper du Canada, Ottawa, Parcs Canada, ministère du Patrimoine, 51 p.

—, 2001-LL. - Lake Louise, Parc National Banff du Canada, Plan Communautaire, Ottawa, ministère du Patrimoine, juin 2001, 32 p.

PARKs Canada, 1994. - Communities (Report), Ottawa, Parks Canada, Department of Canadian Heritage, Operational review \# 29, July 1994, pagination spécifique.

—, 2000. - Wasagaming Community Plan, Ottawa, Parks Canada, Heritage Canada, 43 p.

—, 2004. - Banff National Park of Canada - Management plan: (April 1997) Amended May 2004, Ottawa, Minister of Public Works and Government Services Canada, 111 p.

—, 2005. - State of the Park Report: Jasper National Park of Canada, February 2005, Ottawa, 95 p.

Patrimoine Canadien, 1994. - Principes directeurs et politiques de gestion, Ottawa, Patrimoine canadien, 127 p.

Richez (G.), 1991. - «Aménagement et urbanisation dans les Parcs Nationaux des Rocheuses canadiennes », Études canadiennes/Canadian Studies, n 31, p. 31-48.

—, 1992, Parcs nationaux et tourisme en Europe, Paris, L'Harmattan, 421 p. 
STÉPHANE HÉRITIER

—, 2000a. - « Le Parc National de Banff (Alberta, Canada) face aux défis du tourisme de masse », Études Canadiennes, $\mathrm{n}^{\circ} 49$, p. 145-165.

—, 2000b. - «Politiques publiques et intégrité écologique : L'exemple du Parc national Banff (Alberta, Canada)», Revue de tourisme, n 3, St Gallen (Suisse), p. 21-35.

SalzL (L.), 1984. - The Origin and History of some Places Names in Prince Albert National Park, Waskesiu, Prince Albert National Park, Visitor Service, n.p.

SAnguin (A.-L.), Gill (A.), 1990. - « Le Columbia Icefield et le Glacier Athabasca (Rocheuses Canadiennes), la géomorphologie glaciaire au service du tourisme », Geographica Helvetica, vol. 45, n³ 3, p. 95-104.

—, 1991. - « Le Parc National de Jasper (Alberta), Gestion et aménagement d'un grand espace récréatif des Rocheuses Canadiennes », Revue de Géographie Alpine, vol. 79, n² 2, p. 121-136.

SCACE (R. C.), 1969. - « Banff Townsite: An Historical-Geographical view of Urban Development in a Canadian National Park », p. 187-208, dans Nelson (J. G.), 1969, Canadian Parks in Perspective, Montréal, Harvest House, 343 p.

—, 1979. - « Townsites and other Facilities and Services in Canadian National Parks », p. 529-549, dans Nelson (J. G.), Needham (R. D.), Nelson (S. H.), Scace (R. C.), 1979. - The Canadian National Parks: Today and Tomorrow, Conference II, Ottawa, James Gordon Nelson, volume I : 523 p., volume II, 837 p.

SvateK (G.), 2004. - « Banff lands more than $\$ 8$ million for infracture », Banff Crag $\&$ Canyon, tuesday August 17, 2004, (n.p.).

TAbulenas (D. T.), 1983. - A Narrative Human History of Riding Mountain National Park and Area: Prehistory to 1980, Report prepared for Riding Mountain National Park, $430 \mathrm{p}$.

Tandukar (P.), 1999. - Getting Participation and Keeping Stakeholders involved in Round Table, Report of Internship for Riding Mountain National Park, Canada, 15 p.

Taylor (L.), Ryall (A.), 2003. - Sustainable Mountain Communities: Environmental Sustainability for Mountain Areas Impacted by Tourism and Amenity Migration, Proceedings of a Conference held at the Banff Centre, June 14-18, 2003, 249 p.

Town OF BAnfF, 1998. - Banff Community Plan, 47 p. [plus annexes].

Town of BAnfF InCorporation AgreEment, 1989. - Memorandum of Agreement between the Government of Canada and the Government of Alberta, December 12 $2^{\text {th }}, 1989,37$ p.

Waiser (B.), 1989. - Saskatchewan's Playgrounds : A History of Prince Albert National Park, Saskatoon, Saskatchewan, Fifth South Publishers, 144 p.

Weir (J. M. H.), Pidwerbeski (A.), 2000. - Prince Albert National Park Fire Management Plan, Waskesiu Lake, Resource Conservation Prairie and Northern Region, Parks Canada, April 20, 2000, 20 p.

White (C.), 1985. - Wildland Fires in Banff National Park, 1880-1980, Ottawa, Minister of Supply and Services Canada/National Parks Branch/Parks Canada/Environment Canada, Occasional Paper, n 3, 106 p.

WinKS (Q.), 2005. - « Transferability nearing Reality », Banff Crag E Canyon, Tuesday August 09, 2005, (n.p.).

Cet article a été reçu le 12 septembre 2005 et définitivement accepté le 26 janvier 2006.

\section{REMERCIEMENTS}

Je tiens à adresser mes remerciements sincères pour tous les personnels de Parcs Canada et des municipalités qui ont bien voulu me recevoir et m'accorder de longs entretiens : qu'ils reçoivent ici l'expression de ma gratitude. Par ailleurs, Il m'importe de citer et de remercier plus particulièrement certaines personnes qui ont rendu possible la réalisation de ce travail par leur soutien et leur disponibilité: Gaby Fortin, Directeur régional des parcs de l'Ouest au moment de l'étude, $M^{\text {me }}$ Jillian Roulet, Directeur du Parc National Banff, Cathy Jenkins et Elaine Lemieux (Townsite Manager, PN Yoho), Jim Lamb, chargé de la direction du Parc National Lacs Waterton en 2005, Ifan Thomas (Assistant du Directeur du Parc, Jasper), Megan Squire (Planner, Town of Banff), Greg Fenton, Directeur du Parc National Riding Mountain, Sandy Hicke (Townsite Manager, PN Prince Albert), Murray Peterson (Garde de parc, PN Prince Albert), Sophie Lauro (Assistante de Communication, PN Banff). Enfin, cet article a bénéficié des remarques de Gérard Richez, coordinateur de ce numéro, et des évaluateurs anonymes de la revue Norois. Qu'ils accueillent ces lignes comme l'expression de mes remerciements. 